\title{
Risk factors for secondary hemophagocytic lymphohistiocytosis in severe coronavirus disease 2019 adult patients
}

Mei Meng ${ }^{1,2 \dagger}$, Limin Chen ${ }^{1 \dagger}$, Sheng Zhang ${ }^{1 \dagger}$, Xuan Dong ${ }^{3 \dagger}$, Wenzhe $\mathrm{Li}^{1,2 \dagger}$, Ranran Li $i^{1}$, Yunxin Deng ${ }^{1,2}$, Tao Wang ${ }^{1,2}$,

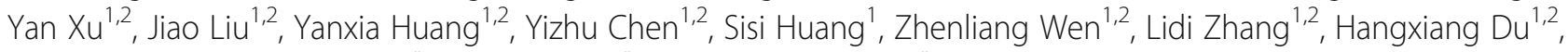
Yongan Liu ${ }^{1,2}$, Djillali Annane ${ }^{4^{*}}$, Jieming $\mathrm{Qu}^{5,6^{*}}$ and Dechang Chen ${ }^{1^{*}}$

\begin{abstract}
Background: Secondary hemophagocytic lymphohistiocytosis (sHLH) is a life-threatening hyperinflammatory event and a fatal complication of viral infections. Whether sHLH may also be observed in patients with a cytokine storm induced by severe acute respiratory syndrome coronavirus 2 (SARS-CoV-2) infection is still uncertain. We aimed to determine the incidence of sHLH in severe COVID-19 patients and evaluate the underlying risk factors.

Method: Four hundred fifteen severe COVID-19 adult patients were retrospectively assessed for hemophagocytosis score (HScore). A subset of 7 patients were unable to be conclusively scored due to insufficient patient data.

Results: In 408 patients, 41 (10.04\%) had an HScore $\geq 169$ and were characterized as "suspected sHLH positive". Compared with patients below a HScore threshold of 98, the suspected sHLH positive group had higher D-dimer, total bilirubin, alanine aminotransferase, aspartate aminotransferase, blood urea nitrogen, serum creatinine, triglycerides, ferritin, interleukin-6, C-reactive protein, procalcitonin, lactate dehydrogenase, creatine kinase isoenzyme, troponin, Sequential Organ Failure Assessment (SOFA) score, while leukocyte, hemoglobin, platelets, lymphocyte, fibrinogen, pre-albumin, albumin levels were significantly lower (all $P<0.05$ ). Multivariable logistic regression revealed that high ferritin $(>1922.58 \mathrm{ng} / \mathrm{mL})$, low platelets $\left(<101 \times 10^{9} / \mathrm{L}\right)$ and high triglycerides $(>2.28$ $\mathrm{mmol} / \mathrm{L}$ ) were independent risk factors for suspected SHLH in COVID-19 patients. Importantly, COVID-19 patients that were suspected sHLH positive had significantly more multi-organ failure. Additionally, a high HScore (>98) was an independent predictor for mortality in COVID-19.
\end{abstract}

(Continued on next page)

\footnotetext{
*Correspondence: djillali.annane@aphp.fr; jmqu0906@163.com;

15168887139@163.com

${ }^{\dagger}$ Mei Meng, Limin Chen, Sheng Zhang, Xuan Dong and Wenzhe Li

contributed equally to this work.

${ }^{4}$ General intensive care unit, Raymond Poincaré Hospital (APHP), Laboratory

of Inflammation and Infection U1173, University of Versailles SQY/INSERM

104 bd Raymond Poincaré, 92380 Garches, France

${ }^{5}$ Department of Pulmonary and Critical Care Medicine, Ruijin Hospital,

Shanghai Jiao Tong University School of Medicine, Shanghai, China

'Department of Critical Care Medicine, Ruijin Hospital, Shanghai Jiao Tong

University School of Medicine, No 197, Rui Jin 2nd road, Shanghai 200025,

China

Full list of author information is available at the end of the article
}

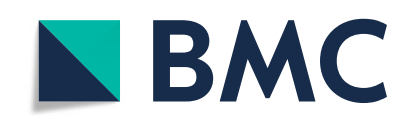

(c) The Author(s). 2021 Open Access This article is licensed under a Creative Commons Attribution 4.0 International License, which permits use, sharing, adaptation, distribution and reproduction in any medium or format, as long as you give appropriate credit to the original author(s) and the source, provide a link to the Creative Commons licence, and indicate if changes were made. The images or other third party material in this article are included in the article's Creative Commons licence, unless indicated otherwise in a credit line to the material. If material is not included in the article's Creative Commons licence and your intended use is not permitted by statutory regulation or exceeds the permitted use, you will need to obtain permission directly from the copyright holder. To view a copy of this licence, visit http://creativecommons.org/licenses/by/4.0/. The Creative Commons Public Domain Dedication waiver (http://creativecommons.org/publicdomain/zero/1.0/) applies to the data made available in this article, unless otherwise stated in a credit line to the data. 
(Continued from previous page)

Conclusions: HScore should be measured as a prognostic biomarker in COVID-19 patients. In particular, it is important that HScore is assessed in patients with high ferritin, triglycerides and low platelets to improve the detection of suspected sHLH.

Keywords: Secondary Hemophagocytic Lymphohistiocytosis, COVID-19, HScore, Ferritin, Triglycerides, Platelet, Cytokine storm

\section{Highlights}

- Secondary hemophagocytic lymphohistiocytosis (sHLH) has prognostic value in corona virus disease 2019 (COVID-19)

- HScore in severe COVID-19 cases can help detect sHLH

- Risk factors for suspected sHLH in severe COVID19 are high ferritin (>1922.58 ng/mL), low platelets $\left(<101 \times 10^{9} / \mathrm{L}\right)$ and high triglycerides $(>2.28 \mathrm{mmol} /$ L).

- Cytokine storm syndrome is the cause of suspected sHLH in severe COVID-19

\section{Introduction}

COVID-19 caused by SARS-CoV-2 continues to spread globally and represents a serious worldwide health problem [1]. RECOVERY trial results showed dexamethasone reduced 28-day mortality in severe COVID-19 by attenuating the exaggerated inflammatory response in the host [2]. However, in coronavirus infection, several studies observed delay in viral clearance with systemic corticosteroid therapy, potentially indicating increased viral replication as an adverse effect [1]. Therefore, it is urgent to accurately identify the patient subsets that may benefit from immunosuppressive treatment.

Accumulating evidence has indicated that a cytokine storm caused by an excessive immune response drives disease progression and organ failure in COVID-19 patients [3-5]. sHLH is a cytokine-driven fulminant hyperinflammatory syndrome associated with morbidity and mortality up to $88 \%$. sHLH in adults often occurs after infections, especially viral infections by Epstein-Barr Virus (EBV), cytomegalovirus (CMV), human immunodeficiency virus (HIV), and influenza [6]. The main clinical features of sHLH include persistent fever, cytopenia, and hyperferritinemia [7]. Lung involvement is common and was shown to be an independent determinant of death in sHLH [8]. It is reasonable to speculate that the abnormal hyperinflammatory state in COVID-19 may induce $\mathrm{SHLH}$, thus driving a poor prognosis.

A recent study suggested that HScore, a score for the diagnosis of reactive hemophagocytic syndrome, could be used to identify suspected occurrence of sHLH in severe COVID-19 without mandatory bone marrow hemophagocytosis [3]. Once a diagnosis of sHLH is established, targeted interventions should be carried out rapidly, including immunosuppressants such as glucocorticoids and chemotherapy drugs [7]. To provide more accurate diagnosis and appropriate treatment, it is necessary to conduct studies about sHLH in severe COVID19.

In this study, we screened severe COVID-19 patients for sHLH with objectives to (1) determine the incidence of suspected sHLH in severe COVID-19, (2) describe clinical features of suspected sHLH associated with COVID-19, (3) evaluate risk factors for suspected sHLH and identify mortality-associated risk factors in COVID19 patients, and (4) highlight potential relationships between emerging knowledge of COVID-19 disease progression and suspected sHLH.

\section{Patients and methods}

The present study is a single-center, retrospective study, which was approved by the ethics committee of Wuhan Infectious Diseases Hospital (KY-2020-03-01).

\section{Data collection}

A total of 1399 confirmed COVID-19 patients from January 2, 2020 to March 28, 2020 were screened. We retrieved demographic and epidemiological characteristics, laboratory testing results, clinical diagnosis and treatment data from electronic medical records and files.

\section{Definition}

Severe COVID-19 was defined according to WHO 2020 classification, as follows: fever or suspected respiratory infection, plus one of the following: respiratory rate $>30$ breaths/min; severe respiratory distress; or $\mathrm{SpO}_{2} \leq 93 \%$ on room air [9]. The Berlin Definition was used to diagnose acute respiratory distress syndrome (ARDS) [10]. Acute kidney injury (AKI) was diagnosed using the KDIGO criteria [11]. Prothrombin time test, the levels of fibrin/fibrinogen (Fib) degradation products, and platelet counts were used for the diagnosis of disseminated intravascular coagulation (DIC) [12]. Diagnosis of septic shock was based on the Third International Consensus Definition for Sepsis and Septic Shock [13]. Liver and heart injury were diagnosed according to laboratory testing results [14]. 
The HScore was developed by Fardet et al. and colleagues to estimate an individual's risk of having sHLH. This scoring system can be freely available online (http://saintantoine.aphp.fr/score/) and contains nine items in routine practice, including known underlying immunodepression, maximal temperature $\left({ }^{\circ} \mathrm{C}\right)$, hepatomegaly, splenomegaly, lower hemoglobin level, lower leucocytes count, lower platelets count, higher ferritin level $(\mathrm{ng} / \mathrm{ml})$, higher triglyceride level $(\mathrm{mmol} / \mathrm{l})$, lower fibrinogen level $(\mathrm{g} / \mathrm{l})$, higher serum glutamic oxaloacetic transaminase/ recombinant aspartate aminotransferase (SGOT/ASAT) level (UI/L), hemophagocytosis features on bone marrow aspirate. We set HScore 169 and 98 as the cut off because HScore at 169 achieved the optimal discriminative power in previous study and HScore $<98$ virtually rules out the diagnosis of sHLH [15]. Patients with HScores between 98 and 169 were not categorized as either suspected sHLH positive or negative [15].

\section{Statistical analysis}

Continuous variables were presented as median (lower quartile, upper quartile) and compared using the Wilcoxon rank-sum or Kruskal-Wallis tests. Frequency was used to represent the unordered classification variables and Pearson chi-square tests were used for comparison. Ordered categorical variables were expressed as frequency and compared by Wilcoxon rank-sum or Kruskal-Wallis tests. A binary logistic regression model was used for multivariate analysis to select factors associated with suspected sHLH or death. The receiveroperator characteristic analysis and area under the curve were used to evaluate the discrimination of potential variables to diagnose suspected sHLH. The model was validated internally by bootstrap resampling, and the prognostic performance was measured by the concordance index (C-index) and calibration curve. All analyses were performed using SAS software, version 9.4 (SAS Institute Inc., Cary, NC) or R software. Graphs were made in Stata 16.0 or MedCalc softwares. All probability calculations were two-tailed, and $p$ values of 0.05 or less were considered to be statistically significant.

\section{Results}

\section{Clinical characteristics and laboratory findings of severe COVID-19}

Of the 1399 COVID-19 confirmed patients who met a treatment endpoint, either recovery or death, in Wuhan Infectious Diseases Hospital by March 28, 2020 were included in this study. Of 1399 patients, 424 met the criteria for severe COVID-19. However, 9 patients were missing, and thus 415 patients were included in this study for analysis. Among the 415 patients, 171 were female $(41.20 \%)$ and 244 were male $(58.80 \%), 255$ were over than 60 years old (61.45\%). Non-survivors were 220 (53.01\%), including 138 male (62.73\%).

Compared with survivors, non-survivors were older and had shorter length of hospital stay, higher SOFA scores and APACHE II scores at $24 \mathrm{~h}$ post-admission, significantly lower leukocytes, platelets, neutrophils, lymphocytes, Fib, pre-albumin, albumin, while D-Dimer, aspartate aminotransferase (AST), blood urea nitrogen (BUN), serum creatinine $(\mathrm{sCr})$, ferritin, Interleukin -6 (IL-6), C-reactive protein (CRP), procalcitonin (PCT), lactate dehydrogenase (LDH), creatine kinase isoenzyme (CK-MB), troponin were all significantly higher (all $P<$ 0.0001) (Table 1). There were no significant differences in underlying comorbidities between survivor and nonsurvivor patients.

\section{Clinical characteristics and laboratory findings of COVID- 19 associated with suspected SHLH according to HScore} Of the 415 patients the available data was only sufficient to generate HScores for 408. Of these 408 patients, 41 (10.05\%) had a HScore $\geq 169$ and were identified as suspected sHLH. Conversely, 279 (68.38\%) had a HScore $\leq 98$ were considered negative for suspected sHLH. The remaining 88 patients had HScores 98-169 and were not categorized.

Mortality in the suspected SHLH positive group was significantly higher than that in the suspected SHLH negative group (100.00\% vs $34.77 \%, P<0.001)$ (Table 2 ).

There were no statistically significant differences in age and gender between suspected sHLH negative and positive groups. Clinical symptoms and signs were similar in suspected sHLH positive group and negative group, except cyanosis was more frequent in suspected sHLH positive patients $(P=0.03)$. For comobidities, suspected sHLH positive patients had significantly higher autoimmue disease $(P=0.042)$.

Compared to the suspected sHLH negative group, the suspected sHLH positive group had significantly higher prevalence of complications including ARDS, DIC, liver injury, heart injury, AKI and, shock as well as high SOFA scores (all $P<0.05$ ) (Table 2).

Several laboratory findings were also statistically different between patients with suspected sHLH compared to those without, such as lower haemoglobin $(\mathrm{Hb})$, platelets, lymphocytes, fibrinogen, pre-albumin, albumin, and higher D-Dimer, total bilirubin (Tbil), alanine aminotransferase (ALT), AST, triglycerides (TG), BUN, sCr, IL-6, PCT, LDH, ferritin, CK-MB, troponin,

With regard to treatments, of 41 suspected sHLH suspected patients, $8.11 \%(n=3)$ received high-flow nasal cannula, $32.43 \%(n=12)$ received non-invasive positivepressure ventilation (NPPV), 37.84\% $(n=14)$ were intubated, $8.11 \%(n=3)$ received extracorporeal membrane oxygenation, $21.95 \%(n=9)$ received continuous renal 
Table 1 Characteristics of all 415 patients with severe COVID-19 and stratified into survivors and non-survivors

\begin{tabular}{|c|c|c|c|c|}
\hline & All $(n=415)$ & Survivors $(n=195)$ & Nonsurvivors $(n=220)$ & $P$ value \\
\hline Gender, Male/Female, n (\%) & $244(58.80) / 171(41.20)$ & $106(54.36) / 89(45.64)$ & $138(62.73) / 82(37.27)$ & 0.0839 \\
\hline Age, years, Mean (SD) & 62.63(13.49) & $57.77(12.67)$ & $66.93(12.74)$ & $<0.0001$ \\
\hline age, years, n(\%) & & & & $<0.0001$ \\
\hline$<40$ & $23(5.54)$ & 15(7.69) & $8(3.64)$ & \\
\hline $40-60$ & 137(33.01) & $92(47.18)$ & $45(20.45)$ & \\
\hline$\geq 60$ & $255(61.45)$ & $88(45.13)$ & 167(75.91) & \\
\hline ARDS, n(\%) & $258(62.93) / 152(37.07)^{a} ; 5$ & 75(38.46) /120(61.54) & 183(85.12)/ 32(14.88); 5 & $<0.0001$ \\
\hline $\mathrm{DIC}, \mathrm{n}(\%)$ & 20(5.00)/380(95.00); 15 & $1(0.52) / 193(99.48) ; 1$ & 19(9.22) /187(90.78); 14 & $<0.0001$ \\
\hline Liver injury, n(\%) & $78(19.50) / 322(80.50) ; 15$ & $26(13.40) / 168(86.60) ; 1$ & $52(25.24) / 154(74.76) ; 14$ & 0.0028 \\
\hline Heart injury, n(\%) & 104(26.00)/296(74.00); 15 & 12(6.19) /182(93.81); 1 & $92(44.66) / 114(55.34) ; 14$ & $<0.0001$ \\
\hline Kidney injury, n(\%) & $60(15.00) / 340(85.00) ; 15$ & $5(2.58) / 189(97.42) ; 1$ & 55(26.70)/ 151(73.30); 14 & $<0.0001$ \\
\hline Shock, n (\%) & $87(21.75) / 313(78.25) ; 15$ & 2(1.03)/ 192(98.97); 1 & $85(41.26) / 121(58.74) ; 14$ & $<0.0001$ \\
\hline Smoking history, n(\%) & 20(4.84)/393(95.16); 2 & 15(7.69)/ 180(92.31) & $5(2.29) / 213(97.71) ; 2$ & 0.0107 \\
\hline Alcohol history, n(\%) & 21(5.08)/ 392(94.92); 2 & 15(7.69)/ 180(92.31) & $6(2.75) / 212(97.25) ; 2$ & 0.0225 \\
\hline \multicolumn{5}{|l|}{ Comobidities } \\
\hline Diabetes, n(\%) & 79(19.13)/334(80.87); 2 & $30(15.38) / 165(84.62)$ & $49(22.48) / 169(77.52) ; 2$ & 0.0673 \\
\hline Hypertension, n(\%) & $147(35.59) / 266(64.41) ; 2$ & $64(32.82) / 131(67.18)$ & $83(38.07) / 135(61.93) ; 2$ & 0.2657 \\
\hline Chronic heart failure, n(\%) & 39(9.44)/374(90.56); 2 & $12(6.15) / 183(93.85)$ & 27(12.39)/ 191(87.61); 2 & 0.0306 \\
\hline COPD, n(\%) & 13(3.15) /400(96.85); 2 & $3(1.54) / 192(98.46)$ & 10(4.59)/ 208(95.41); 2 & 0.0765 \\
\hline Stroke, $n(\%)$ & $26(6.30) / 387(93.70) ; 2$ & 10(5.13)/ 185(94.87) & 16(7.34)/ 202(92.66); 2 & 0.3557 \\
\hline Malignant Tumor, n(\%) & $19(4.60) / 394(95.40) ; 2$ & $10(5.13) / 185(94.87)$ & $9(4.13) / 209(95.87) ; 2$ & 0.6283 \\
\hline Chronic liver disease, n(\%) & 14(3.39)/ 399(96.61); 2 & $5(2.56) / 190(97.44)$ & $9(4.13) / 209(95.87) ; 2$ & 0.3805 \\
\hline Chronic kidey disease, n(\%) & $11(2.66) / 402(97.34) ; 2$ & $3(1.54) / 192(98.46)$ & $8(3.67) / 210(96.33) ; 2$ & 0.1793 \\
\hline Hepatitis B, n(\%) & 15(3.62)/399(96.38); 1 & 7(3.59)/ 188(96.41) & $8(3.65) / 211(96.35) ; 1$ & 0.9726 \\
\hline HIV, n (\%) & $0(0.00) / 414(100.00) ; 1$ & $0(0.00) / 195(100.00)$ & $0(0.00) / 219(100.00) ; 1$ & 1.0000 \\
\hline Autoimmue disease, $\mathrm{n}(\%)$ & $17(4.12) / 396(95.88) ; 2$ & 7(3.59)/ 188(96.41) & 10(4.59)/ 208(95.41); 2 & 0.6105 \\
\hline Respiratory support, n(\%) & & & & $<0.0001$ \\
\hline Intranasal oxygen inhalation & 146(37.15) & $122(63.21)$ & $24(12.00)$ & \\
\hline Mask oxygen Inhalation & $18(4.58)$ & 10(5.18) & $8(4.00)$ & \\
\hline High Flow & $67(17.05)$ & $24(12.44)$ & $43(21.50)$ & \\
\hline Non-invasive ventilation & $75(19.08)$ & $9(4.66)$ & $66(33.00)$ & \\
\hline Invasive ventilaiton & $53(13.49)$ & $3(1.55)$ & $50(25.00)$ & \\
\hline ECMO & $4(1.02)$ & $0(0.00)$ & $4(2.00)$ & \\
\hline CRRT, n(\%) & 79(19.13)/334(80.87); 2 & $30(15.38) / / 165(84.62)$ & 49(22.48)/ 169(77.52); 2 & 0.0673 \\
\hline Inotropic support, n(\%) & $87(20.96) / 328(79.03)$ & $2(1.03) / 193(98.97)$ & $85(38.64) / 135(61.36)$ & $<0.0001$ \\
\hline Corticosteroid treatment, $\mathrm{n}(\%)$ & $226(54.45) / 189(45.54)$ & $85(43.59) / 110(56.41)$ & $141(64.09) / 79(35.91)$ & $<0.0001$ \\
\hline \multicolumn{5}{|l|}{ Co-infection, n(\%) } \\
\hline Influenza A & $401(97.33) / 6(1.46) ; 8$ & 193(98.97)/ 2(1.03) & 208(95.85)/ 4(1.84); 8 & 0.0799 \\
\hline Influenza B & $402(97.57) / 5(1.21) ; 8$ & 195(100.00)/ O(0.00) & $207(95.39) / 5(2.30) ; 8$ & 0.0101 \\
\hline Tuberculosis & $8(1.94) / 405(98.06) ; 2$ & $3(1.54) / 192(98.46)$ & $5(2.29) / 213(97.71) ; 2$ & 0.8428 \\
\hline \multicolumn{5}{|l|}{ Symptoms and signs, $\mathrm{n}(\%)$} \\
\hline heart rate, (per minute) & & & & 0.0002 \\
\hline$\leq 100$ & $37(15.42)$ & $28(26.17)$ & $9(6.77)$ & \\
\hline$>100$ & 200(83.33) & $77(71.96)$ & 123(92.48) & \\
\hline
\end{tabular}


Table 1 Characteristics of all 415 patients with severe COVID-19 and stratified into survivors and non-survivors (Continued)

\begin{tabular}{|c|c|c|c|c|}
\hline & All $(n=415)$ & Survivors( $n=195)$ & Nonsurvivors $(n=220)$ & $P$ value \\
\hline Total & $240(100.00)$ & 107(100.00) & 133(100.00) & \\
\hline Nasal stuffiness & $8(1.94) / 405(98.06) ; 2$ & $4(2.05) / 191(97.95)$ & $4(1.83) / 214(98.17) ; 2$ & 1.0000 \\
\hline Nasal discharge & 12(2.91) /401(97.09); 2 & $5(2.56) / 190(97.44)$ & 7(3.21)/ 211(96.79); 2 & 0.6960 \\
\hline Sneezing & $4(0.97) / 408(99.03) ; 3$ & $3(1.54) / 192(98.46)$ & $1(0.46) / 216(99.54) ; 3$ & 0.5414 \\
\hline Sore throat & $8(1.94) / 405(98.06) ; 2$ & $4(2.05) / 191(97.95)$ & $4(1.83) / 214(98.17) ; 2$ & 1.0000 \\
\hline Cough & $330(79.90) / 83(20.10) ; 2$ & 157(80.51)/ 38(19.49) & 173(79.36)/ 45(20.64); 2 & 0.7700 \\
\hline Sputum production & $157(38.01) / 256(61.99) ; 2$ & $67(34.36) / 128(65.64)$ & $90(41.28) / 128(58.72) ; 2$ & 0.1478 \\
\hline Chest tightness & 264(63.92)/ 149(36.08); 2 & $113(57.95) / 82(42.05)$ & 151(69.27)/ 67(30.73); 2 & 0.0168 \\
\hline Chest pain & 12(2.91)/401(97.09); 2 & 6(3.08)/ 189(96.92) & $6(2.75) / 212(97.25) ; 2$ & 0.8445 \\
\hline Hemoptysis & $7(1.69) / 406(98.31) ; 2$ & $4(2.05) / 191(97.95)$ & $3(1.38) / 215(98.62) ; 2$ & 0.8817 \\
\hline Headache & $13(3.15) / 400(96.85) ; 2$ & 10(5.13)/ 185(94.87) & $3(1.38) / 215(98.62) ; 2$ & 0.0292 \\
\hline Myalgia & $49(11.86) / 364(88.14) ; 2$ & 29(14.87)/ 166(85.13) & 20(9.17)/ 198(90.83); 2 & 0.0739 \\
\hline Fatigue & $165(40.05) / 247(59.95) ; 3$ & 78(40.21)/ 116(59.79) & 87(39.91)/ 131(60.09); 2 & 0.9509 \\
\hline Digestive symptoms & $58(14.04) / 355(85.96) ; 2$ & 28(14.36)/ 167(85.64) & $30(13.76) / 188(86.24) ; 2$ & 0.8615 \\
\hline Discomfort of eye & 1(0.24)/ 412(99.76); 2 & $1(0.51) / 194(99.49)$ & $0(0.00) / 218(100.00) ; 2$ & 0.4722 \\
\hline Cyanosis & $48(11.59) / 366(88.41) ; 1$ & 10(5.13)/ 185(94.87) & $38(17.35) / 181(82.65) ; 1$ & 0.0001 \\
\hline Rhonchial & $26(6.30) / 386(93.46) ; 3$ & $13(6.67) / 181(92.82)$ & 13(5.96)/ 205(94.04); 2 & 1.0000 \\
\hline Moist rales & $76(18.40) / 337(81.60) ; 2$ & 33(16.92)/ 162(83.08) & 43(19.72)/ 175(80.28); 2 & 0.4632 \\
\hline $\mathrm{SPO}_{2}(\leq 93 \% />93 \%)$ & $279(67.23) / 136(32.77)$ & $96(49.23) / 99(50.77)$ & 183(83.18)/ 37(16.82) & $<0.0001$ \\
\hline Length of hospitalization, $d$ & 12.00(7.00,18.00); & $15.00(11.00,20.00)$ & $10.00(4.00,15.00)$ & $<0.0001$ \\
\hline Length of ICU, d & $2.00(0.00,9.00)$ & $0.00(0.00,6.50)$ & $3.00(1.00,9.00)$ & $<0.0001$ \\
\hline Temperature, ${ }^{\circ} \mathrm{C}$ & $38.50(38.00,39.00)$ & $38.50(38.00,39.00)$ & $38.50(38.00,39.00)$ & 0.5281 \\
\hline arterial pressure, mmHg & 87.00(79.83,92.83); 21 & $86.00(78.00,91.33) ; 2$ & $88.00(82.00,93.67) ; 19$ & 0.0005 \\
\hline heart rate, per mimute & $89.00(81.00,100.00) ; 2$ & $90.00(82.00,100.00)$ & $88.50(80.00,102.00) ; 2$ & 0.9356 \\
\hline respiratory rate & $22.00(20.00,26.00) ; 1$ & $22.00(20.00,26.00)$ & $22.00(20.00,28.00)$ & 0.7331 \\
\hline$<24$, (per minute) & $251(60.48)$ & $122(62.56)$ & 129(58.64) & 0.3642 \\
\hline 24-30, (per minute) & $87(20.96)$ & $40(20.51)$ & $47(21.36)$ & \\
\hline$\geq 30$, (per minute) & $77(18.55)$ & $33(16.92)$ & $44(20.00)$ & \\
\hline Total & $415(100.00)$ & 195(100.00) & $220(100.00)$ & \\
\hline SOFA & $3.00(1.00,4.00) ; 4$ & $2.00(1.00,3.00)$ & $3.00(2.00,5.00) ; 4$ & $<0.0001$ \\
\hline APACHEII & $7.00(5.00,10.00) ; 4$ & $6.00(4.00,7.00)$ & $9.00(7.00,13.00) ; 4$ & $<0.0001$ \\
\hline \multicolumn{5}{|l|}{ Laboratory Tests } \\
\hline Lowest leukocyte, $\times 10^{9} / \mathrm{L}$ & $5.44(3.83,8.50)^{\mathrm{b}} ; 10$ & $4.45(3.43,5.91)$ & $7.57(4.63,11.60) ; 10$ & $<0.0001$ \\
\hline Lowest haemoglobin, g/L & $109.00(88.00,123.00) ; 10$ & $110.00(91.00,124.00)$ & $108.00(86.00,122.00) ; 10$ & 0.5267 \\
\hline Lowest patelets, $\times 10^{9} / \mathrm{L}$ & $125.50(53.00,188.00) ; 9$ & $152.00(86.00,217.00)$ & $91.00(42.00,165.00) ; 9$ & $<0.0001$ \\
\hline Lowest neutrophils, $\times 10^{9} / \mathrm{L}$ & $5.38(3.00,11.36) ; 14$ & $3.55(2.55,8.59)$ & $6.99(4.30,11.63) ; 14$ & $<0.0001$ \\
\hline Lowest lymphocyte, $\times 10^{9} / \mathrm{L}$ & $0.55(0.35,1.06) ; 11$ & $0.86(0.49,1.44)$ & $0.44(0.28,0.64) ; 11$ & $<0.0001$ \\
\hline Lowest fibrinogen, $g / L$ & $4.10(2.70,5.90) ; 22$ & $4.50(3.40,6.50) ; 6$ & $3.60(2.20,5.30) ; 16$ & $<0.0001$ \\
\hline Lowest prealbumin, g/L & $83.00(45.00,117.00) ; 10$ & $104.00(67.00,145.00) ; 1$ & $64.00(33.00,98.00) ; 9$ & $<0.0001$ \\
\hline Lowest albumin, g/L & $28.00(25.10,31.40) ; 8$ & $30.10(27.60,34.40)$ & $26.00(23.45,28.70) ; 8$ & $<0.0001$ \\
\hline Peak D-Dimer, mg/L & $10.06(1.15,42.82)$ & $1.58(0.63,14.04)$ & $27.31(7.60,58.76)$ & $<0.0001$ \\
\hline Peak total bilirubin, $\mu \mathrm{g} / \mathrm{L}$ & 18.95(13.00,32.30); 13 & $15.40(11.70,30.00) ; 2$ & $22.50(15.39,32.76) ; 11$ & 0.0019 \\
\hline Peak alanine aminotransferase, $\mathrm{U} / \mathrm{L}$ & 49.00(31.00,77.00); 12 & $48.00(30.00,68.00)$ & $50.50(31.50,87.00) ; 12$ & 0.1652 \\
\hline Peak aspartate aminotransferase, U/L & $43.00(29.00,72.00) ; 11$ & $34.00(24.00,50.00)$ & $57.00(36.00,108.00) ; 11$ & $<0.0001$ \\
\hline
\end{tabular}


Table 1 Characteristics of all 415 patients with severe COVID-19 and stratified into survivors and non-survivors (Continued)

\begin{tabular}{|c|c|c|c|c|}
\hline & All $(n=415)$ & Survivors $(n=195)$ & Nonsurvivors $(n=220)$ & $P$ value \\
\hline Peak blood urea nitrogen, $\mathrm{mmol} / \mathrm{L}$ & $9.20(5.97,18.00) ; 14$ & $6.66(5.03,9.79) ; 1$ & $14.90(8.10,24.30) ; 13$ & $<0.0001$ \\
\hline Peak serum creatinine, $\mu \mathrm{g} / \mathrm{L}$ & $82.00(59.40,139.80) ; 13$ & $70.70(53.70,89.30)$ & $120.70(69.30,276.80) ; 13$ & $<0.0001$ \\
\hline Peak triglycerides, $\mathrm{mmol} / \mathrm{L}$ & $2.15(1.42,3.43) ; 14$ & $2.16(1.42,4.53) ; 1$ & $2.10(1.41,3.27) ; 13$ & 0.5062 \\
\hline Peak ferritin, $\mu \mathrm{g} / \mathrm{L}$ & 927.22(492.02,2000.00); 18 & $600.08(356.00,987.50) ; 6$ & $1675.09(837.84,2000.00) ; 12$ & $<0.0001$ \\
\hline Peak Interleukin $-6, \mathrm{pg} / \mathrm{mL}$ & $11.18(7.92,19.57) ; 26$ & $9.32(6.90,13.50) ; 9$ & $13.89(9.99,27.90) ; 17$ & $<0.0001$ \\
\hline Peak erythrocyte sedimentation rate, $\mathrm{mm} / \mathrm{h}$ & $60.00(43.00,79.00) ; 22$ & $61.00(45.30,88.00) ; 4$ & $59.35(40.00,73.00) ; 18$ & 0.0527 \\
\hline Peak C-reactive protein, mg/L & $122.60(30.50,160.00) ; 12$ & $49.40(8.70,121.90) ; 3$ & $160.00(111.00,160.40) ; 9$ & $<0.0001$ \\
\hline Peak procalcitonin, $\mathrm{ng} / \mathrm{mL}$ & $0.20(0.06,2.87) ; 17$ & $0.07(0.05,0.23) ; 4$ & $1.08(0.16,6.40) ; 13$ & $<0.0001$ \\
\hline Peak lactate dehydrogenase, U/L & $429.00(274.00,699.00) ; 10$ & $300.50(221.00,403.00) ; 1$ & $675.00(464.00,1095.00) ; 9$ & $<0.0001$ \\
\hline Peak creatine kinase isoenzyme, $U / L$ & $27.00(17.00,66.00) ; 16$ & $19.50(14.00,51.00) ; 1$ & $34.00(20.00,84.00) ; 15$ & $<0.0001$ \\
\hline Peak Troponin, pg/mL & $10.85(3.50,99.00) ; 13$ & $4.40(1.70,9.10) ; 1$ & $70.50(11.20,823.05) ; 12$ & $<0.0001$ \\
\hline
\end{tabular}

Abbreviations: ARDS Acute Respiratory Distress Syndrome, DIC Disseminated Intravascular Coagulation, COPD Chronic Obstructive Pulmonary Disease, ECMO Extracorporeal Membrane Oxygenation, CRRT Continuous Renal Replacement Therapy, ICU Intensive Care Unit, SOFA Sequential Organ Failure Assessment, APACHE Acute Physiology, Age, Chronic Health Evaluation

Data are expressee as: ${ }^{\mathrm{a}}$ No. (\%) yes/no; no. missing (if applicable); ${ }^{\mathrm{b}}$ Median (range); no. missing (if applicable)

$P$ value compare between survivor group vs non-survivor group, $P<0.05$ means had significantly different

replacement therapy $(\mathrm{CRRT}), 51.22 \%(n=21)$ received inotropic support and $85.37 \%(n=35)$ received corticosteroid treatment. Except CRRT $(P=0.56)$, all abovementioned treatments in suspected sHLH positive group were significantly more common compared to suspected sHLH negative group (all $P<0.05$ ) (Table 2 ).

\section{Risk factors for mortality of severe COVID-19}

High SOFA score, low leukocyte, low lymphocyte, low prealbumin, high AST, high CRP, high LDH and old age, were all significantly associated with death in severe COVID-19 patients by logistic regression analysis (all $P<0.05$ ) (Table 3). In addition a higher HScore was also significantly associated with death in severe COVID-19 patients. This association was significant in comparison of patients with HScore $\leq 98$ versus patients with HScore $\geq 169$ (Odds Ratio $=22.77$, 95\% Confidence Interval, 2.23-232.80, $P=0.008$ ), and was also significant with comparison of patients with HScore $\leq 98$ versus patients with HScore 98-169 (Odds Ratio = 4.02, 95\% Confidence Interval, $1.55-10.44, P=0.004$ ).

\section{Risk factors related to COVID-19 associated suspected sHLH}

Multivariable logistic regression analyses found that independent risk factors for discriminating suspected sHLH in COVID-19 included high triglycerides (Odds Ratio, 0.74; 9\% Confidence Interval, 0.61-0.90), high ferritin (Odds Ratio, 0.99; 95\% Confidence Interval, 0.9970.999), low platelets (Odds Ratio, 1.0079; 95\% Confidence Interval, 1.00-1.01) (Table 4).

Receiver operating characteristic curve analysis indicated that high ferritin may alone diagnose suspected sHLH in COVID-19, which had an area under the curve
(AUC) of $0.816(P<0.01$, sensitivity $85.4 \%$, specificity $76.6 \%$, cut-off value $>1922.58 \mathrm{ng} / \mathrm{mL}$ ). A combination of high ferritin (cut-off value $>1922.58 \mathrm{ng} / \mathrm{mL}$ ), high triglycerides (cut-off value $>2.28 \mathrm{mmol} / \mathrm{L}$ ), and low platelets (cut-off value $<101 \times 10^{9} / \mathrm{L}$ ) had an AUC of 0.863 $(P<0.001$, sensitivity $85.4 \%$, specificity $75.4 \%)$ (Fig. 1 ).

Internal validation was conducted using a bootstrapping strategy (1000 repetitions), the results achieved a C-index of 0.863 (95\%CI $0.838-0.889)$. The calibration plot was shown Fig. 2.

\section{Discussion}

Herein, we present data that demonstrates the prognostic value of HScores, and three independent risk factors for high HScores in a specific cohort of severe COVID19 patients. In this cohort, mortality was $53.01 \%$ in patients with severe COVID-19 and 100\% in patients with suspected sHLH. Furthermore, we observed a significantly higher incidence of multi-organ dysfunction in patients with suspected sHLH. The frequency rate of suspected sHLH in severe COVID-19 patients was $10.05 \%$ (41 of 408). We assessed the clinical factors associated with suspected sHLH including hemoglobin, platelets, lymphocytes, fibrinogen, pre-albumin, albumin, D-dimer, total bilirubin, blood urea nitrogen, serum creatinine, triglycerides, ferritin, interleukin-6, C-reactive protein, pro-calcitonin, lactate dehydrogenase, creatinine kinase-MB and troponin. The independent risk factors of suspected $\mathrm{sHLH}$ in this study were low platelet $\operatorname{count}\left(<101 \times 10^{9} / \mathrm{ml}\right)$, high triglycerides $(>2.28 \mathrm{mmol} / \mathrm{L})$ and high ferritin $(>1922.58 \mathrm{ng} / \mathrm{mL})$.

There are two major kinds of HLH: primary and secondary. Primary HLH is related to gene defects wherein typically CD8 and Natural Killer (NK) mediated 
Table 2 Clinical Characteristics and Laboratory Findings of COVID-19 according to HScore ( $\leq 98$ as sHLH negative, $\geq 169$ as sHLH positive, 98-169 as uncertain)

\begin{tabular}{|c|c|c|c|c|}
\hline & $\leq 98(n=279)$ & $\geq 169(n=41)$ & $98-169(n=88)$ & $P$ value \\
\hline outcome & & & & $<0.0001$ \\
\hline Survivor, n(\%) & 182(65.23) & $0(0.00)$ & $13(14.77)$ & \\
\hline Non-survivor, n(\%) & $97(34.77)$ & $41(100.00)$ & $75(85.23)$ & \\
\hline Total & $279(100.00)$ & $41(100.00)$ & $88(100.00)$ & \\
\hline Gender, Male/Female, n(\%) & 153(54.84)/ 126(45.16) & $27(65.85) / 14(34.15)$ & $60(68.18) / 28(31.82)$ & 0.0537 \\
\hline Age, years, Mean (SD) & $63.00(52.00,72.00)$ & $63.00(52.00,71.00)$ & $65.00(57.00,72.00)$ & 0.2419 \\
\hline age, years, n(\%) & & & & 0.1869 \\
\hline$<40$ & $17(6.09)$ & $3(7.32)$ & $3(3.41)$ & \\
\hline $40-60$ & $101(36.20)$ & $11(26.83)$ & $25(28.41)$ & \\
\hline$\geq 60$ & $161(57.71)$ & $27(65.85)$ & $60(68.18)$ & \\
\hline ARDS, n(\%) & $150(53.76) / 129(46.24)^{a}$ & $34(85.00) / 6(15.00) ; 1$ & 68(80.95)/16(19.05); 4 & $<0.0001$ \\
\hline DIC, n(\%) & $6(2.17) / 271(97.83) ; 2$ & $4(10.53) / 34(89.47) ; 3$ & $10(12.66) / 69(87.34) ; 9$ & 0.0004 \\
\hline Liver, n(\%) & $36(13.00) / 241(87.00) ; 2$ & $12(31.58) / 26(68.42) ; 3$ & 30(37.97)/49(62.03); 9 & $<0.0001$ \\
\hline Heart, n(\%) & $50(18.05) / 227(81.95) ; 2$ & $17(44.74) / 21(55.26) ; 3$ & 35(44.30)/44(55.70); 9 & $<0.0001$ \\
\hline Kidney, n(\%) & $17(6.14) / 260(93.86) ; 2$ & $16(42.11) / 22(57.89) ; 3$ & $26(32.91) / 53(67.09) ; 9$ & $<0.0001$ \\
\hline Shock, n(\%) & $31(11.19) / 246(88.81) ; 2$ & $20(52.63) / 18(47.37) ; 3$ & $34(43.04) / 45(56.96) ; 9$ & $<0.0001$ \\
\hline Smoking history, n(\%) & 14(5.04)/264(94.96); 1 & $2(4.88) / 39(95.12)$ & $4(4.60) / 83(95.40) ; 1$ & 0.9111 \\
\hline Alcohol history, n(\%) & 16(5.76)/262(94.24); 1 & $1(2.44) / 40(97.56)$ & $4(4.60) / 83(95.40) ; 1$ & 0.3577 \\
\hline \multicolumn{5}{|l|}{ Comobidities } \\
\hline Diabetes,n(\%), n(\%) & $50(17.99) / 228(82.01) ; 1$ & $9(21.95) / 32(78.05)$ & $20(22.73) / 68(77.27)$ & 0.5630 \\
\hline Hypertension, n(\%) & $97(34.89) / 181(65.11) ; 1$ & $11(26.83) / 30(73.17)$ & $38(43.18) / 50(56.82)$ & 0.1639 \\
\hline Chronic heart failure, n(\%) & 29(10.43)/249(89.57); 1 & $2(4.88) / 39(95.12)$ & $7(7.95) / 81(92.05)$ & 0.2138 \\
\hline COPD, n(\%) & $5(1.80) / 273(98.20) ; 1$ & $2(4.88) / 39(95.12)$ & $6(6.82) / 82(93.18)$ & 0.0537 \\
\hline Stroke, $n(\%)$ & 18(6.47)/260(93.53); 1 & $0(0.00) / 41(100.00)$ & $8(9.09) / 80(90.91)$ & 0.3853 \\
\hline Tumor, n(\%) & 11(3.96)/267(96.04); 1 & $2(4.88) / 39(95.12)$ & $5(5.68) / 83(94.32)$ & 0.5927 \\
\hline Chronic liver disease, n(\%) & $6(2.16) / 272(97.84) ; 1$ & 2(4.88)/39(95.12) & $6(6.82) / 82(93.18)$ & 0.0907 \\
\hline Chronic kidey disease, n(\%) & $7(2.52) / 271(97.48) ; 1$ & $2(4.88) / 39(95.12)$ & $2(2.27) / 86(97.73)$ & 0.5200 \\
\hline Hepatitis B,n(\%) & 11(3.94)/268(96.06) & $3(7.32) / 38(92.68)$ & 1(1.15)/86(98.85); 1 & 0.7610 \\
\hline HIV, n(\%) & $0(0.00) / 279(100.00)$ & $0(0.00) / 41(100.00)$ & $0(0.00) / 87(100.00) ; 1$ & 1.0000 \\
\hline Autoimmue disease, n(\%) & $7(2.52) / 271(97.48) ; 1$ & $3(7.32) / 38(92.68)$ & $6(6.82) / 82(93.18)$ & 0.0422 \\
\hline Respiratory support, n(\%) & & & & $<0.0001$ \\
\hline Intranasal oxygen inhalation & $129(47.43)$ & $3(8.11)$ & 13(16.46) & \\
\hline Mask oxygen Inhalation & $16(5.88)$ & $1(2.70)$ & $1(1.27)$ & \\
\hline High Flow & $48(17.65)$ & $3(8.11)$ & 14(17.72) & \\
\hline Non-invasive ventilation & $35(12.87)$ & $12(32.43)$ & $26(32.91)$ & \\
\hline Invasive ventilaiton & $17(6.25)$ & 14(37.84) & $22(27.85)$ & \\
\hline ECMO & $1(0.37)$ & $3(8.11)$ & $0(0.00)$ & \\
\hline CRRT, n(\%) & $50(17.99) / 228(82.01) ; 1$ & $9(21.95) / 32(78.05)$ & $20(22.73) / 68(77.27)$ & 0.5630 \\
\hline Inotropic support, n(\%) & $33(11.83) / 246(88.17)$ & $21(51.22) / 20(48.78)$ & $30(34.09) / 58(65.91)$ & $<0.0001$ \\
\hline Corticosteroid treatment, $\mathrm{n}(\%)$ & 133(47.67)/ 146(52.33) & 35(85.37)/ 6(14.63) & $55(62.50) / 33(37.50)$ & $<0.0001$ \\
\hline \multicolumn{5}{|l|}{ Co-infection, n(\%) } \\
\hline Influenza A & $3(1.08) / 273(98.56) ; 3$ & 1(2.44)/ 38(92.68); 2 & $2(2.27) / 84(95.45) ; 2$ & 0.0924 \\
\hline Influenza B & $1(0.36) / 275(99.28) ; 3$ & $1(2.44) / 38(92.68) ; 2$ & $3(3.41) / 83(94.32) ; 2$ & 0.0127 \\
\hline
\end{tabular}


Table 2 Clinical Characteristics and Laboratory Findings of COVID-19 according to HScore ( $\leq 98$ as sHLH negative, $\geq 169$ as sHLH positive, 98-169 as uncertain) (Continued)

\begin{tabular}{|c|c|c|c|c|}
\hline & $\leq 98(n=279)$ & $\geq 169(n=41)$ & $98-169(n=88)$ & $P$ value $^{c}$ \\
\hline Tuberculosis & $4(1.44) / 274(98.56) ; 1$ & $1(2.44) / 40(97.56)$ & $3(3.41) / 85(96.59)$ & 1.0000 \\
\hline \multicolumn{5}{|l|}{ Symptoms and signs, n(\%) } \\
\hline heart rate, (per minute) & & & & 0.6759 \\
\hline$\leq 100$ & $32(21.77)$ & $2(3.57)$ & $4(6.67)$ & \\
\hline$>100$ & 113(76.87) & $26(92.86)$ & $56(93.33)$ & \\
\hline Total & 147(100.00) & $28(100.00)$ & $60(100.00)$ & \\
\hline Nasal stuffiness & $5(1.80) / 273(98.20) ; 1$ & $2(4.88) / 39(95.12)$ & $1(1.14) 87(98.86)$ & 1.0000 \\
\hline Nasal discharge & $6(2.16) / 272(97.84) ; 1$ & $5(12.20) / 36(87.80)$ & $1(1.14) / 87(98.86)$ & 0.0086 \\
\hline Sneezing & $3(1.08) / 275(98.92) ; 1$ & $0(0.00) / 40(100.00) ; 1$ & $1(1.14) / 87(98.86)$ & 1.0000 \\
\hline Sore throat & $4(1.44) / 274(98.56) ; 1$ & $1(2.44) / 40(97.56)$ & $3(3.41) / 85(96.59)$ & 1.0000 \\
\hline Cough & 226(81.29)/ 52(18.71); 1 & $30(73.17) / 11(26.83)$ & 69(78.41)/ 19(21.59) & 0.4468 \\
\hline Sputum production & 104(37.41)/174(62.59); 1 & 16(39.02)/25(60.98) & $33(37.50) / 55(62.50)$ & 0.9802 \\
\hline Chest tightness & $172(61.87) / 106(38.13) ; 1$ & $30(73.17) / 11(26.83)$ & $58(65.91) / 30(34.09)$ & 0.3367 \\
\hline Chest pain & $7(2.52) / 271(97.48)$ & $1(2.44) / 40(97.56)$ & $4(4.55) / 84(95.45)$ & 0.6647 \\
\hline Hemoptysis & $5(1.80)$ 273(98.20); 1 & $2(4.88) / 39(95.12)$ & $0(0.00) / 88(100.00)$ & 1.0000 \\
\hline Headache & $9(3.24) / 269(96.76) ; 1$ & $2(4.88) / 39(95.12)$ & $2(2.27) / 86(97.73)$ & 0.8098 \\
\hline Myalgia & $34(12.23) / 244(87.77) ; 1$ & $3(7.32) / 38(92.68)$ & $12(13.64) / 76(86.36)$ & 0.5736 \\
\hline Acratia & 102(36.82)/175(63.18);2 & $26(63.41) / 15(36.59)$ & $35(39.77) / 53(60.23)$ & 0.0052 \\
\hline Digestive symptoms & $36(12.95) / 242(87.05)$ & $9(21.95) / 32(78.05)$ & $13(14.77) / 75(85.23)$ & 0.3021 \\
\hline Discomfort of eye & 1(0.36)/ 277(99.64); 1 & $0(0.00) / 41(100.00)$ & $0(0.00) / 88(100.00)$ & 1.0000 \\
\hline Cyanosis & 27(9.71) /251(90.29); 1 & 10(24.39) /31(75.61) & $7(7.95) / 81(92.05)$ & 0.0393 \\
\hline Rhonchial & 18(6.47) 259(93.17); 1 & $4(9.76) / 37(90.24)$ & $4(4.55) / 84(95.45)$ & 1.0000 \\
\hline Moist rales & $51(18.35) / 227(81.65)$ & 12(29.27) /29(70.73) & $12(13.64) 76(86.36)$ & 0.1028 \\
\hline $\mathrm{SPO}_{2}(\leq 93 \% />93 \%)$ & 173(62.01)/ 106(37.99) & $32(78.05) / 9(21.95)$ & $67(76.14) / 21(23.86)$ & 0.0131 \\
\hline Length of hospitalization, $d$ & $12.00(8.00,18.00)^{b}$ & $17.00(11.00,22.50)$ & $12.00(7.00,16.00)$ & 0.0029 \\
\hline Length of ICU, $d$ & $0.00(0.00,6.00)$ & $9.50(3.50,12.00)$ & $3.00(0.50,12.00)$ & $<0.0001$ \\
\hline Temperature, ${ }^{\circ} \mathrm{C}$ & $38.50(38.00,39.00)$ & $38.50(38.00,38.90)$ & $38.90(38.00,39.00)$ & 0.4406 \\
\hline Arterial pressure, $\mathrm{mmHg}$ & $87.00(78.33,93.00)$ & $86.58(81.50,91.33)$ & $86.75(81.33,93.50)$ & 0.1593 \\
\hline Heart rate, per mimute & 89.00(82.00,99.00); 1 & $90.0081 .00,100.00$ & $88.00(80.00,102.00)$ & 0.5895 \\
\hline Respiratory rate & & & & 0.5070 \\
\hline$<24$, (per minute) & 164(58.78) & $26(63.41)$ & $58(65.91)$ & \\
\hline 24-30, (per minute) & 65(23.30) & $5(12.20)$ & $15(17.05)$ & \\
\hline$\geq 30$, (per minute) & $50(17.92)$ & 10(24.39) & 15(17.05) & \\
\hline Total & $279(100.00)$ & $41(100.00)$ & $88(100.00)$ & \\
\hline SOFA & $2.00(1.00,3.00)$ & $3.00(1.00,4.00)$ & $3.00(2.00,5.00)$ & $<0.0001$ \\
\hline APACHEII & $7.00(5.00,9.00)$ & $8.00(6.00,11.00)$ & $8.00(6.00,13.00)$ & 0.0010 \\
\hline \multicolumn{5}{|l|}{ Laboratory Tests } \\
\hline Lowest leukocyte, $\times 10^{9} / \mathrm{L}$ & $5.08(3.74,7.83)^{\mathrm{b}} ; 4$ & $6.97(4.38,9.31)$ & $6.77(3.81,10.53) ; 1$ & 0.0118 \\
\hline Lowest haemoglobin, g/L & $112.00(94.00,124.00) ; 4$ & $95.00(77.00,106.00)$ & 106.00(78.00,122.00); 1 & 0.0020 \\
\hline Lowest patelets, $\times 10^{9} / \mathrm{L}$ & $151.00(81.00,197.00) ; 3$ & $61.00(27.00,101.00)$ & $83.00(26.00,144.00) ; 1$ & $<0.0001$ \\
\hline Lowest neutrophils, $\times 10^{9} / \mathrm{L}$ & $4.73(2.76,11.46) ; 8$ & $5.10(3.32,7.39)$ & $7.02(3.97,12.72) ; 1$ & 0.0804 \\
\hline Lowest lymphocyte, $\times 10^{9} / \mathrm{L}$ & $0.69(0.44,1.22) ; 5$ & $0.33(0.18,0.53)$ & $0.43(0.28,0.66) ; 1$ & $<0.0001$ \\
\hline Lowest fibrinogen, g/L & $4.50(3.24,6.35) ; 15$ & $2.40(1.60,3.46)$ & $3.80(2.10,5.40) ; 2$ & $<0.0001$ \\
\hline
\end{tabular}


Table 2 Clinical Characteristics and Laboratory Findings of COVID-19 according to HScore ( $\leq 98$ as sHLH negative, $\geq 169$ as sHLH positive, 98-169 as uncertain) (Continued)

\begin{tabular}{|c|c|c|c|c|}
\hline & $\leq 98(n=279)$ & $\geq 169(n=41)$ & $98-169(n=88)$ & $P$ value \\
\hline Lowest prealbumin, $\mathrm{g} / \mathrm{L}$ & $93.00(59.00,127.00) ; 4$ & $51.00(28.00,85.00)$ & $55.00(29.00,89.00) ; 1$ & $<0.0001$ \\
\hline Lowest albumin, g/L & $28.90(26.20,32.30) ; 2$ & $24.40(22.30,27.60)$ & $26.60(23.40,28.90) ; 1$ & $<0.0001$ \\
\hline Peak D-Dimer, mg/L & $4.58(0.80,32.09) ; 5$ & $47.82(20.06,80.00)$ & $17.83(3.46,63.70) ; 1$ & $<0.0001$ \\
\hline Peak total bilirubin, $\mu \mathrm{g} / \mathrm{L}$ & $16.60(12.20,30.00) ; 6$ & $23.98(17.38,37.80)$ & $25.20(16.60,32.30) ; 1$ & 0.0007 \\
\hline Peak alanine aminotransferase, U/L & $47.00(28.00,67.00) ; 6$ & $57.00(36.00,122.00)$ & $62.00(38.00,109.00) ; 1$ & 0.0001 \\
\hline Peak aspartate aminotransferase, U/L & $38.00(26.00,55.00) ; 5$ & $85.00(52.00,215.00)$ & $65.00(40.00,121.00) ; 1$ & $<0.0001$ \\
\hline Peak blood urea nitrogen, $\mathrm{mmol} / \mathrm{L}$ & $7.50(5.30,11.00) ; 8$ & $21.66(13.50,35.30)$ & $14.90(8.50,24.00) ; 1$ & $<0.0001$ \\
\hline Peak serum creatinine, $\mu \mathrm{g} / \mathrm{L}$ & $72.95(55.60,99.00) ; 7$ & $246.10(146.00,413.70)$ & 105.20(67.40,263.10); 1 & $<0.0001$ \\
\hline Peak triglycerides, $\mathrm{mmol} / \mathrm{L}$ & $1.95(1.31,3.27) ; 8$ & $3.27(2.33,4.27)$ & $2.20(1.58,3.43) ; 1$ & 0.0001 \\
\hline Peak ferritin, $\mu \mathrm{g} / \mathrm{L}$ & $710.11(433.23,1276.37) ; 11$ & $2000.00(2000.00,2000.00)$ & $2000.00(962.51,2000.00) ; 2$ & $<0.0001$ \\
\hline Peak Interleukin -6, pg/mL & 10.20(7.26,14.98); 16 & $23.41(11.73,60.57) ; 1$ & $14.60(8.56,26.90) ; 4$ & $<0.0001$ \\
\hline Peak erythrocyte sedimentation rate, $\mathrm{mm} / \mathrm{h}$ & $60.00(43.00,80.00) ; 10$ & $65.50(49.20,79.00) ; 3$ & $58.30(39.00,74.00) ; 4$ & 0.5133 \\
\hline Peak C-reactive protein, mg/L & $76.68(15.60,160.00) ; 5$ & $160.00(160.00,206.40)$ & $160.00(121.20,198.80) ; 2$ & $<0.0001$ \\
\hline Peak procalcitonin, $\mathrm{ng} / \mathrm{mL}$ & $0.11(0.05,0.50) ; 10$ & $4.21(2.63,15.22)$ & $0.98(0.19,4.90) ; 2$ & $<0.0001$ \\
\hline Peak lactate dehydrogenase, $\mathrm{U} / \mathrm{L}$ & $349.00(241.00,534.00) ; 4$ & $1096.00(708.00,1676.00)$ & $662.00(432.00,944.00) ; 1$ & $<0.0001$ \\
\hline Peak creatine kinase isoenzyme, $U / L$ & $21.50(15.00,52.50) ; 9$ & $47.00(32.00,85.00)$ & $38.00(21.00,77.00) ; 1$ & $<0.0001$ \\
\hline Peak Troponin, pg/mL & $6.15(2.40,24.60) ; 7$ & $303.10(33.80,2202.10)$ & $51.50(6.44,591.40) ; 1$ & $<0.0001$ \\
\hline
\end{tabular}

Abbreviations: ARDS Acute Respiratory Distress Syndrome, DIC Disseminated Intravascular Coagulation, COPD Chronic Obstructive Pulmonary Disease, ECMO Extracorporeal Membrane Oxygenation, CRRT Continuous Renal Replacement Therapy, ICU Intensive Care Unit, SOFA Sequential Organ Failure Assessment, APACHE Acute Physiology, Age, Chronic Health Evaluation

Data are expressee as: ${ }^{\mathrm{a}} \mathrm{No}$. (\%) yes/no; no. missing (if applicable); ${ }^{\mathrm{b}}$ Median (range); no. missing (if applicable)

${ }^{c} P$ value compare between Hscore $\geq 169$ group vs Hscore $\leq 98$ group, $P<0.05$ means had significantly different

cytotoxic genes are defective. Secondary HLH is acquired through infection, malignant conditions, and autoimmune diseases. It is a rare life-threatening complication characterized by uncontrolled activation of CD8 + T cells and NK cells, cytokine storm, and severe organ dysfunction [6, 7]. However, suspected sHLH lacks specific clinical characters [7]. Our study found that severe COVID-19 patients with suspected sHLH had similar clinical manifestations including body temperature, heart rhythm, respiration, and clinical

Table 3 Logistic regression analyses of potential risk factors of Hemophagocytic lymphohistiosytosis (HLH)

\begin{tabular}{|c|c|c|c|c|}
\hline & Regression Coefficient & OR (Odds Ratio) & $95 \% \mathrm{Cl}$ & $P$ value \\
\hline SOFA & 0.221 & 1.247 & $1.010-1.539$ & 0.040 \\
\hline Lowest haemoglobin, g/L & 0.003 & 1.003 & $0.983-1.023$ & 0.769 \\
\hline Lowest platelet, $\times 10^{9} / \mathrm{L}$ & 0.008 & 1.008 & $1.000-1.015$ & 0.038 \\
\hline Lowest neutrophils, $\times 10^{9} / \mathrm{L}$ & 0.025 & 1.026 & $0.987-1.066$ & 0.195 \\
\hline Lowest fibrinogen, g/L & 0.042 & 1.043 & $0.999-1.089$ & 0.052 \\
\hline Lowest prealbumin, $\mathrm{g} / \mathrm{L}$ & 0.006 & 1.006 & $0.994-1.017$ & 0.332 \\
\hline Peak D-Dimer, mg/L & -0.004 & 0.996 & $0.993-1.00$ & 0.061 \\
\hline Peak aspartate aminotransferase, U/L & -0.000 & 1.000 & $0.999-1.001$ & 0.910 \\
\hline Peak serum creatinine, $\mu \mathrm{g} / \mathrm{L}$ & -0.002 & 0.998 & $0.996-1.000$ & 0.126 \\
\hline Peak triglycerides, $\mathrm{mmol} / \mathrm{L}$ & -0.300 & 0.741 & $0.611-0.900$ & 0.003 \\
\hline Peak ferritin, ng/mL & -0.002 & 0.998 & $0.997-0.999$ & $<0.001$ \\
\hline Peak Interleukin -6, pg/mL & -0.000 & 1.000 & $0.999-1.000$ & 0.175 \\
\hline Peak C-reactive protein, mg/L & -0.002 & 0.998 & $0.990-1.005$ & 0.510 \\
\hline Peak lactate dehydrogenase, U/L & -0.000 & 1.000 & $0.999-1.000$ & 0.639 \\
\hline
\end{tabular}

Abbreviations: SOFA Sequential Organ Failure Assessment

$P$ value compare between Hscore $\geq 169$ group vs Hscore $<169$ group, $P<0.05$ means had significantly different 
Table 4 Logistic regression analyses of potential risk factors of mortality in severe COVID-19 patients

\begin{tabular}{|c|c|c|c|c|}
\hline & Regression Coefficient & OR (Odds Ratio) & $95 \% \mathrm{Cl}$ & $P$ value \\
\hline SOFA & 0.317 & 1.373 & $1.006-1.712$ & 0.005 \\
\hline Lowest leukocyte, $\times 10^{9} / \mathrm{L}$ & 0.277 & 1.319 & $1.174-1.491$ & $<0.001$ \\
\hline Lowest neutrophils, $\times 10^{9} / \mathrm{L}$ & -0.011 & 0.989 & $0.976-1.001$ & 0.080 \\
\hline Lowest lymphocyte, $\times 10^{9} / \mathrm{L}$ & 0.316 & 1.372 & $1.089-1.729$ & 0.007 \\
\hline Lowest prealbumin, g/L & -0.012 & 0.987 & $0.979-0.994$ & 0.001 \\
\hline Lowest albumin, g/L & 0.012 & 1.013 & $0.988-1.038$ & 0.300 \\
\hline Peak D-Dimer, mg/L & -0.001 & 0.998 & $0.991-1.005$ & 0.632 \\
\hline Peak aspartate aminotransferase, U/L & -0.004 & 0.995 & $0.992-0.997$ & 0.000 \\
\hline Peak blood urea nitrogen, mmol/L & 0.0004 & 1.000 & $0.989-1.011$ & 0.931 \\
\hline Peak serum creatinine, $\mu \mathrm{g} / \mathrm{L}$ & 0.001 & 1.001 & $0.999-1.003$ & 0.131 \\
\hline Peak Interleukin -6, pg/mL & -0.000 & 0.999 & $0.998-1.000$ & 0.880 \\
\hline Peak C-reactive protein, mg/L & 0.006 & 1.006 & $1.000-1.012$ & 0.036 \\
\hline Peak lactate dehydrogenase, U/L & 0.003 & 1.003 & $1.001-1.005$ & $<0.001$ \\
\hline Peak creatine kinase isoenzyme, $\mathrm{U} / \mathrm{L}$ & 0.003 & 1.003 & $0.999-1.008$ & 0.080 \\
\hline Peak Troponin, pg/mL & 0.000 & 0.999 & $0.999-1.000$ & 0.293 \\
\hline Age, y & 0.057 & 1.059 & $1.030-1.088$ & $<0.001$ \\
\hline Hscore $98-169$ vs $\leq 98$ & 1.393 & 4.028 & $1.553-10.444$ & 0.004 \\
\hline Hscore $\geq 169$ vs $\leq 98$ & 3.125 & 22.770 & $2.227-232.800$ & 0.008 \\
\hline
\end{tabular}

Abbreviations: SOFA Sequential Organ Failure Assessment

$P$ value compare between survivor group vs nonsurvivor group, $P<0.05$ means had significantly different

symptoms as those without suspected sHLH. The overlap of clinical features impedes the diagnosis of suspected $\mathrm{SHLH}$ in severe infectious diseases especially in the case of cytopenias, prolonged fevers, and nonresponsiveness to treatment $[6,7]$. According to recommendations for the management of hemophagocytic lymphohistiocytosis in adults, reevaluation of the clinical condition should be frequent (at least every $12 \mathrm{~h}$ ) to determine if additional HLH-directed therapy is needed [16]. Therefore, the assessment of HScore in severe COVID-19 patients could improve the detection rates of suspected sHLH and promote early targeted-treatment. The HLH-2004 protocol was also widely used for the diagnosis of hemophagocytic lymphohistiocytosis. We did not apply HLH-2004 as diagnosis tool because most of the patients in our study did not have data on four diagnostic items of HLH-2004 protocol, including haemophagocytosis in bone marrow or spleen or lymph nodes, low or absent natural killer cell activity, soluble CD25 (soluble interleukin-2 receptor).

Progression of COVID-19 into organ failure results from a catastrophic overreaction of the immune system to the virus, known as a "cytokine storm". Cytokine storms are characterized by high levels of IL-6, Creactive protein and ferritin and causes immune cellmediated tissue damage and catastrophic organ failure $[6,7]$. As demonstrated in this study, the levels of IL-6, $\mathrm{C}$-reactive protein, and ferritin were significantly higher in the suspected sHLH positive group than in the suspected sHLH negative group. It has been previously suggested that the presence of high ferritin syndrome in COVID-19 was a high-risk factor for suspected sHLH $[3,5]$. Consistent with this, we observed that increased peak ferritin was associated with suspected $\mathrm{sHLH}$ and with increased fatality in severe COVID-19 $(P<0.001)$. It is important to consider that a higher ferritin level than $1922 \mathrm{ng} / \mathrm{ml}$ may be used as a screening indicator for suspected sHLH related to COVID-19.

Hypertriglyceridemia (fasting, equal to, or greater than $1.5 \mathrm{mmol} / \mathrm{L}$ ) is one of the current diagnostic criteria for suspected sHLH [15]. Our results showed that hypertriglyceridemia $(>2.28 \mathrm{mmol} / \mathrm{L})$ might be an independent risk factor to determine severe COVID19 associated suspected sHLH. It also is easily available and standardized as laboratory diagnostic testing in clinical practice. The hyperinflammatory response contributes to hypertriglyceridemia via prolonged and intense T-lymphocyte and macrophage signaling. The proinflammatory signaling induces elevated IL-6 which mobilizes free fatty acids from adipocytes, decreased lipoprotein lipase activity and increased tumor necrosis factor alpha expression [17]. Furthermore, metabolic syndrome, wherein hypertriglyceridemia is a hallmark, also has characteristic increased serum ferritin levels, suggesting similar pathogenic mechanisms [18]. 
a

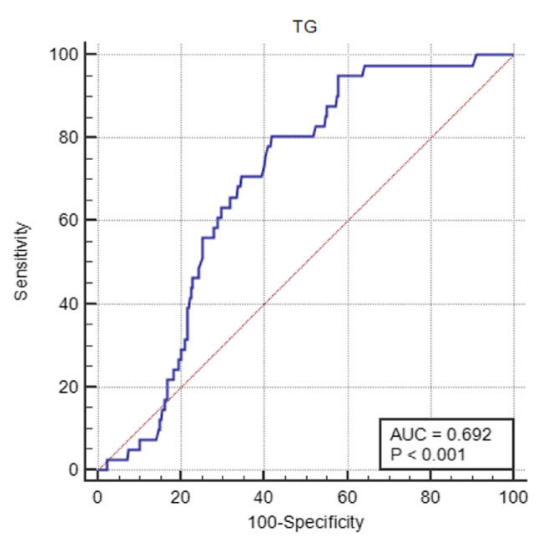

C

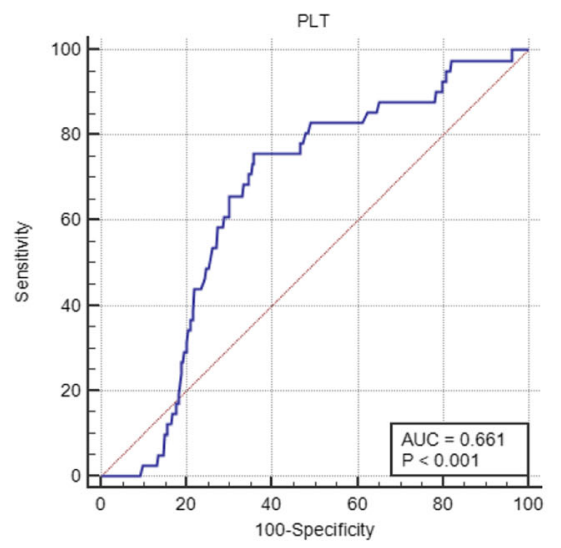

b

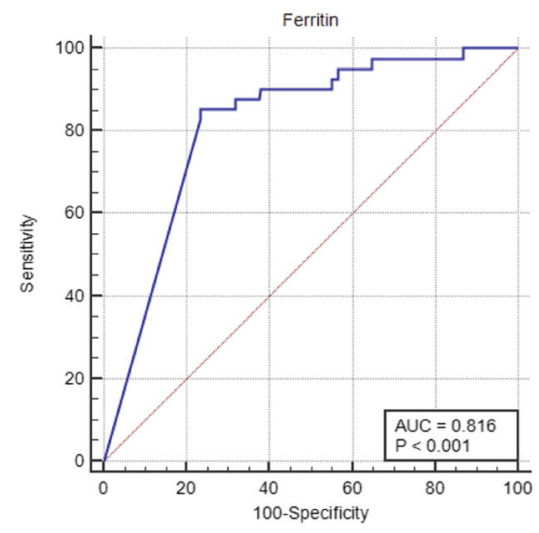

d

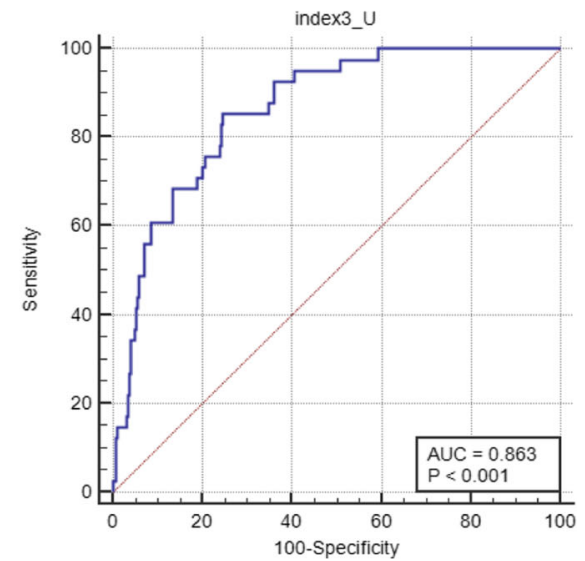

Fig. 1 Receiver operating characteristic curve for peak TG (a), ferritin (b), PLT (c) respectively. Receiver operating characteristic curve for the multivariable logistic regression model, including TG, ferritin, PLT (d). Abbreviations: AUC, area under the curve; TG, triglyceride; PLT, platelet

Thrombocytopenia is reported to be common in COVID-19 patients and is considered a risk factor for increased disease severity and mortality [19]. In our cohort study, severe COVID-19 patients had a low median platelet count $\left(125.50 \times 10^{9} / \mathrm{L}\right)$. The mechanism underlying the low count of platelet in COVID-19 is multifactorial and complex. First, SARS-CoV-2 damages endothelial cells triggering platelet activation and thrombosis in multi-organ, causing vast platelet consumption $[19,20]$. Second, excessive inflammation in COVID-19 may induce venous and arterial thromboembolism. The incidence of thrombus in ICU patients with COVID-19 was found to be as high as $31 \%$ [21]. Moreover, suspected sHLH leads to depletion of blood cells and can cause cytopenia such as thrombocytopenia [6]. Therefore, we expected that platelets would be much lower in suspected sHLH patients. In the present study, we observed that platelet count, a simple and readily available biomarker, lower than $101 \times 10^{9} / \mathrm{L}$ is an independent risk factor and can be used to differentiate patients with suspected sHLH in this severe COVID-19 cohort. Thus, high ferritin, hypertriglyceridemia and low platelets are predictive of sHLH and should be measured in patients with severe COVID-19, to identify and facilitate specific treatment of sHLH. In addition, the prediction value of "peak TG, ferritin, and low PLT" in COVID-19 patients were supported by a series of previous studies [22-24].

We found that 41 patients who fulfilled suspected sHLH probability diagnosis according to HScore succumbed to COVID-19, while suspected SHLH negative patients had a survival rate of $68.38 \%$. One of the possible reasons for the high mortality is the lack of targeted-treatment for suspected sHLH. Adult HLH patients with dyspnea, diarrhea, diffuse bleeding, jaundice and septicemia accompanied by organ failure were characterized by extreme symptoms of severe suspected sHLH, which has very poor prognosis [25]. These signs and symptoms, along with significant disease progression, provide a risk signature for possible HLH, consistent with the clinical manifestations of COVID-19 with 


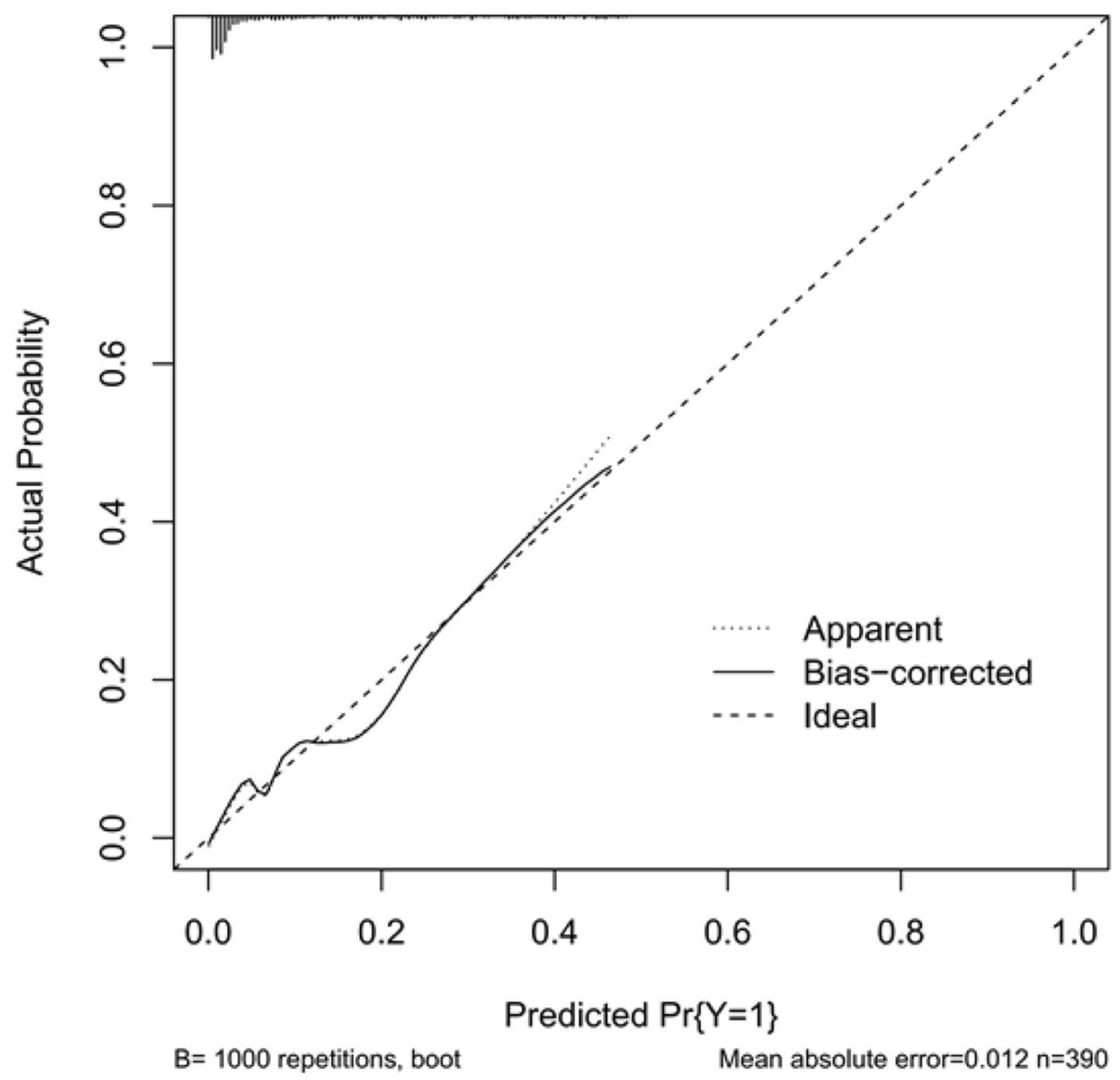

Fig. 2 Internal validation to validate a prediction model

cytokine storm [26]. Recently research indicated that suspected sHLH related to COVID-19 received IL-6 antagonistic therapy and Anakinra (recombinant soluble receptor antagonist of IL-1 $\beta$ and IL-1 $\alpha$ ) [27, 28]. Several drugs targeting specific cytokines are in on-going clinical trials in patients with COVID-19 [29] and the earliest possible detection of sHLH is of the utmost importance.

Our study has several limitations. First, in the severe COVID-19 group, some cases of suspected sHLH may have been missed, suggesting that suspected sHLH may have been underestimated in this study. The lack of definite indicators in the 88 patients with HScore ranging between 98 and 169 could have led to the underestimation of suspected sHLH. Second, all patients lacked specific diagnostic indicators for sHLH, such as laboratory test assessing soluble CD25 and NK-cell activity due to insufficient medical resources to provide these tests during the COVID-19 outbreak. As a retrospective study, it is difficult to infer a definite causality between sHLH and severe COVID-19. Therefore, we selected HScores $\geq 169$ as the positive cut-off value and HScores $\leq 98$ as the negative cut-off value to prevent overestimating suspected sHLH in these patients. Third, the upper limit of detection for ferritin was $2000 \mathrm{ng} / \mathrm{ml}$, which might reduce the specificity of ferritin as a predictor of suspected sHLH. Fourth, the clinical parameters of the liver/spleen and cytological results of bone marrow aspiration were virtually absent in all patients. Fifth, there is no second validation cohort for the prediction model. In order to overcome the question, internal validation was conducted using bootstrapping (1000 repetitions), the results achieved a C-index $0.863(95 \%$ CI $0.838-0.889)$.

\section{Conclusion}

Although HScore has been widely used in various infectious diseases to identify $\mathrm{sHLH}$, the criteria were not specifically studied for use in COVID-19 patients. It is important to find the clinical markers that predict mortality in COVID-19 patients. We recommend HScore for early suspected sHLH screening in severe COVID-19 patients with high ferritin, high triglyceride and low platelet levels. Prospective studies are needed to inform whether it is possible to utilize these markers to determine suspected sHLH diagnosis to identify at-risk populations to target for HLH therapy in severe COVID-19 in the future. 


\section{Abbreviations}

sHLH: Secondary hemophagocytic lymphohistiocytosis; SARS-CoV-2: Severe acute respiratory syndrome coronavirus 2; COVID-19: Corona virus disease 2019; HScore: Hemophagocytosis score; EBV: Epstein-Barr Virus:

CMV: Cytomegalovirus; HIV: Human immunodeficiency virus;

SOFA: Sequential Organ Failure Assessment; ARDS: Acute respiratory distress syndrome; AKI: Acute kidney injury; Fib, fibrin/fibrinogen; DIC: Disseminated intravascular coagulation; SGOT: Serum glutamic oxaloacetic transaminase; ASAT: Recombinant aspartate aminotransferase; APACHE II: Acute Physiology and Chronic Health Evaluation II; AST: Aspartate aminotransferase;

BUN: Blood urea nitrogen; sCr: Serum creatinine; IL-6: Interleukin - 6; CRP: Creactive protein; PCT: Procalcitonin; LDH: Lactate dehydrogenase; CKMB: Creatine kinase isoenzyme; Hb: Haemoglobin; Tbil, total bilirubin; ALT: Alanine aminotransferase; TG: Triglycerides; NPPV: Non-invasive positivepressure ventilation; CRRT: Continuous renal replacement therapy; AUC: Area under the curve; C-index: Concordance index; NK: Natural Killer

\section{Acknowledgments}

We thank all the healthcare workers who have been fighting against COVID19 at the frontline. We would like to express our appreciation for all the patients included in this study as well as their families. We also would like to thank Wuhan Infectious Disease Hospital and all the staff members that have participated in the treatment of COVID-19 patients.

We express deep appreciation to Coopersmith CM, MD (Director of Emory Critical Center, Emory University School of Medicine, Atlanta, GA) who edited this manuscript.

We would like to thank Editage (www.editage.cn) for English language editing.

\section{Authors' contributions}

MM, LMC and SZ were responsible for statistical analysis and figures construction. MM, XD, WZL and RRL wrote this manuscript and organized tables and figures. YXD, TW, YX, JL, YXH, YZC, SSH, ZLW and LDZ were responsible for medical information collection. HXD, YAL, YZC, SSH, ZLW and LDZ were responsible for data extraction and verification. AD, JMQ, and DCC designed and guided this study. All authors reviewed and approved the final version.

\section{Funding}

The study was supported by grants from the Shanghai Science and Technology Commission, China (No. 20Y11901700, No. 20DZ2200500) and a grant from the Shandong Science and Technology Commission, China (No. ZR2019MH016). The funding bodies had no role in the design of the study, data collection, data analysis and interpretation, and in writing the manuscript.

\section{Availability of data and materials}

The data that support the findings of this study are available from the corresponding author on reasonable request. Participant data without names and identifiers will be made available after approval from the corresponding author. After publication of study findings, the data will be available for others to request. The research team will provide an email address for communication once the data are approved to be shared with others.

\section{Declarations}

\section{Ethics approval and consent to participate}

The ethics committee of Wuhan Infectious Diseases Hospital granted a waiver of informed consent from study participants (KY-2020-03-01). The data used in this study was anonymised before its use.

\section{Consent for publication}

Not applicable. No individual person's data was shown separately. All data are presented as a whole.

\section{Competing interests}

The authors declare no competing interests.

\section{Author details}

'Department of Critical Care Medicine, Ruijin Hospital, Shanghai Jiao Tong University School of Medicine, No 197, Rui Jin 2nd road, Shanghai 200025,
China. ${ }^{2}$ Department of Critical Care Medicine, Ruijin Hospital North, Shanghai Jiao Tong University School of Medicine, Shanghai, China. ${ }^{3}$ Tuberculosis and Respiratory Department, Wuhan Infectious Diseases Hospital, Wuhan, China. ${ }^{4}$ General intensive care unit, Raymond Poincaré Hospital (APHP), Laboratory of Inflammation and Infection U1173, University of Versailles SQY/INSERM 104 bd Raymond Poincaré, 92380 Garches, France. ${ }^{5}$ Department of Pulmonary and Critical Care Medicine, Ruijin Hospital, Shanghai Jiao Tong University School of Medicine, Shanghai, China. ${ }^{6}$ Institute of Respiratory Diseases, Shanghai Jiao Tong University School of Medicine, Rui Jin 2nd road, Shanghai 200025, China.

Received: 18 October 2020 Accepted: 20 April 2021 Published online: 29 April 2021

\section{References}

1. Alhazzani W, Møller MH, Arabi YM, Loeb M, Gong MN, Fan E, et al. Surviving Sepsis campaign: guidelines on the management of critically ill adults with coronavirus disease 2019 (COVID-19). Intensive Care Med. 2020;46(5):854-87. https://doi.org/10.1007/s00134-020-06022-5.

2. Horby P, Lim WS, Emberson J, Mafham M, Bell J, Linsell L, et al. Effect of Dexamethasone in Hospitalized Patients with COVID-19: Preliminary Report 2020.

3. Mehta P, McAuley DF, Brown M, Sanchez E, Tattersall RS, Manson JJ. COVID19: consider cytokine storm syndromes and immunosuppression. Lancet. 2020;395(10229):1033-4. https://doi.org/10.1016/S0140-6736(20)30628-0.

4. Huang C, Wang Y, Li X, Ren L, Zhao J, Hu Y, et al. Clinical features of patients infected with 2019 novel coronavirus in Wuhan, China. Lancet. 2020;395(10223):497-506. https://doi.org/10.1016/S0140-6736(20)30183-5.

5. Colafrancesco S, Alessandri C, Conti F, Priori R. COVID-19 gone bad: a new character in the spectrum of the hyperferritinemic syndrome? Autoimmun Rev. 2020;19(7):102573. https://doi.org/10.1016/j.autrev.2020.102573.

6. Al-Samkari H, Berliner N. Hemophagocytic Lymphohistiocytosis. Annu Rev Pathol. 2018;13(1):27-49. https://doi.org/10.1146/annurev-pathol-020117-043 625.

7. Ramos-Casals M, Brito-Zerón P, López-Guillermo A, Khamashta MA, Bosch X. Adult haemophagocytic syndrome. Lancet. 2014;383(9927):1503-16. https:// doi.org/10.1016/S0140-6736(13)61048-X.

8. Seguin A, Galicier L, Boutboul D, Lemiale V, Azoulay E. Pulmonary involvement in patients with Hemophagocytic Lymphohistiocytosis. Chest. 2016;149(5):1294-301. https://doi.org/10.1016/j.chest.2015.11.004.

9. World Health Organization. Clinical management of severe acute respiratory infection (SARI) when COVID-19 disease is suspected. https://www.who.int/ docs/default-source/coronaviruse/clinical-management-of-novel-cov. pdf?ffvrsn=bc7da517_10\&download=true. Accessed 14 Mar 2020.

10. Ranieri VM, Rubenfeld GD, Thompson BT, Ferguson ND, Caldwell E, Fan E, et al. Acute respiratory distress syndrome: the Berlin definition. JAMA. 2012; 307(23):2526-33. https://doi.org/10.1001/jama.2012.5669.

11. Khwaja A. KDIGO clinical practice guidelines for acute kidney injury. Nephron Clin Pract. 2012;120(4):c179-84. https://doi.org/10.1159/000339789.

12. Touaoussa A, El Youssi H, El Hassani I, Hanouf D, El Bergui I, Zoulati G, et al. Disseminated intravascular coagulation: clinical and biological diagnosis. Ann Biol Clin. 2015;73(6):657-63. https://doi.org/10.1684/abc.2015.1100.

13. Singer M, Deutschman CS, Seymour CW, Shankar-Hari M, Annane D, Bauer $M$, et al. The third international consensus definitions for Sepsis and septic shock (Sepsis-3). JAMA. 2016;315(8):801-10. https://doi.org/10.1001/jama.201 6.0287 .

14. Dunn GD, Hayes P, Breen KJ, Schenker S. The liver in congestive heart failure: a review. Am J Med Sci. 1973;265(3):174-89. https://doi.org/10.1097/ 00000441-197303000-00001.

15. Fardet L, Galicier L, Lambotte O, Marzac C, Aumont C, Chahwan D, et al. Development and validation of the HScore, a score for the diagnosis of reactive hemophagocytic syndrome. Arthritis Rheumatol. 2014;66(9):261320. https://doi.org/10.1002/art.38690.

16. La Rosée P, Horne A, Hines M, von Bahr GT, Machowicz R, Berliner N, et al. Recommendations for the management of hemophagocytic lymphohistiocytosis in adults. Blood. 2019;133(23):2465-77. https://doi.org/1 $0.1182 /$ blood.2018894618.

17. Khovidhunkit W, Memon RA, Feingold KR, Grunfeld C. Infection and inflammation-induced proatherogenic changes of lipoproteins. J Infect Dis. 2000;181(Suppl 3):S462-72. https://doi.org/10.1086/315611. 
18. Mateo-Gallego R, Lacalle L, Pérez-Calahorra S, Marco-Benedí V, Recasens V, Padrón $\mathrm{N}$, et al. Efficacy of repeated phlebotomies in hypertriglyceridemia and iron overload: a prospective, randomized, controlled trial. J Clin Lipidol. 2018;12(5):1190-8. https://doi.org/10.1016/j.jacl.2018.06.017.

19. Lippi G, Plebani M, Henry BM. Thrombocytopenia is associated with severe coronavirus disease 2019 (COVID-19) infections: a meta-analysis. Clin Chim Acta. 2020;506:145-8. https://doi.org/10.1016/j.cca.2020.03.022.

20. Varga Z, Flammer AJ, Steiger P, Haberecker M, Andermatt R, Zinkernagel AS, et al. Endothelial cell infection and endotheliitis in COVID-19. Lancet. 2020; 395(10234):1417-8. https://doi.org/10.1016/S0140-6736(20)30937-5.

21. Klok FA, Kruip M, van der Meer NJM, Arbous MS, Gommers D, Kant KM, et al. Incidence of thrombotic complications in critically ill ICU patients with COVID-19. Thromb Res. 2020;191:145-7. https://doi.org/10.1016/j.thromres.2 020.04.013.

22. Ruan Q, Yang K, Wang W, Jiang L, Song J. Clinical predictors of mortality due to COVID-19 based on an analysis of data of 150 patients from Wuhan, China. Intensive Care Med. 2020; published online March 3. https://doi.org/1 0.1007/s00134-020-05991-X.

23. Retamozo S, Brito-Zerón P, Sisó-Almirall A, Flores-Chávez A, Soto-Cárdenas M, Ramos-Casals M. Haemophagocytic syndrome and COVID-19. Clin Rheumatol. 2021;3(4):1-12. https://doi.org/10.1007/s10067-020-05569-4.

24. Mousavi SA, Rad S, Rostami T, Rostami M, Mousavi S, Mirhoseini S, et al. Hematologic predictors of mortality in hospitalized patients with COVID-19: a comparative study. Hematology. 2020;25(1):383-8. https://doi.org/10.1 080/16078454.2020.1833435.

25. McGonagle D, Sharif K, O'Regan A, Bridgewood C. The role of cytokines including Interleukin-6 in COVID-19 induced pneumonia and macrophage activation syndrome-like disease. Autoimmun Rev. 2020;19(6):102537. https://doi.org/10.1016/j.autrev.2020.102537.

26. England JT, Abdulla A, Biggs CM, Lee AYY, Hay KA, Hoiland RL, et al. Weathering the COVID-19 storm: lessons from hematologic cytokine syndromes. Blood Rev. 2021;45:100707. https://doi.org/10.1016/j.blre.2020.1 00707.

27. Radbel J, Narayanan N, Bhatt PJ. Use of Tocilizumab for COVID-19-induced cytokine release syndrome: a cautionary case report. Chest. 2020;158(1):e159. https://doi.org/10.1016/j.chest.2020.04.024.

28. Dimopoulos G, de Mast Q, Markou N, Theodorakopoulou M, Komnos A Mouktaroudi M, et al. Favorable Anakinra Responses in Severe Covid-19 Patients with Secondary Hemophagocytic Lymphohistiocytosis. Cell Host Microbe. 2020;28(1):117-123.e1.

29. Henderson LA, Canna SW, Schulert GS, Volpi S, Lee PY, Kernan KF, et al. On the alert for cytokine storm: immunopathology in COVID-19. Arthritis Rheumatol. 2020;72(7):1059-63. https://doi.org/10.1002/art.41285.

\section{Publisher's Note}

Springer Nature remains neutral with regard to jurisdictional claims in published maps and institutional affiliations.

Ready to submit your research? Choose BMC and benefit from:

- fast, convenient online submission

- thorough peer review by experienced researchers in your field

- rapid publication on acceptance

- support for research data, including large and complex data types

- gold Open Access which fosters wider collaboration and increased citations

- maximum visibility for your research: over $100 \mathrm{M}$ website views per year

At BMC, research is always in progress.

Learn more biomedcentral.com/submissions 